\title{
Belgeo
}

Revue belge de géographie

$1-2 \mid 2010$

Villes et grands équipements de transport/Cities and large transport infrastructures

\section{Désirs de gares TGV : du projet des édiles locaux au "désaménagement" du territoire}

Desire for stations : from local officials" projects to territorial "displanning"

Jean-François Troin

\section{CpenEdition}

Journals

Édition électronique

URL : http://journals.openedition.org/belgeo/6647

DOI : $10.4000 /$ belgeo.6647

ISSN : 2294-9135

Éditeur :

National Committee of Geography of Belgium, Société Royale Belge de Géographie

Édition imprimée

Date de publication : 30 septembre 2010

Pagination : 23-34

ISSN : $1377-2368$

Référence électronique

Jean-François Troin, « Désirs de gares TGV : du projet des édiles locaux au "désaménagement" du territoire », Belgeo [En ligne], 1-2 | 2010, mis en ligne le 05 décembre 2012, consulté le 19 avril 2019. URL : http://journals.openedition.org/belgeo/6647; DOI : 10.4000/belgeo.6647

Ce document a été généré automatiquement le 19 avril 2019

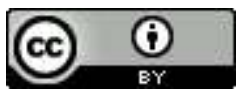

Belgeo est mis à disposition selon les termes de la licence Creative Commons Attribution 4.0 International. 


\section{Désirs de gares TGV : du projet des édiles locaux au "désaménagement" du territoire}

Desire for stations : from local officials' projects to territorial "displanning"

Jean-François Troin

\section{Introduction}

1 Il y avait un tramway nommé désir, il y a aujourd'hui des gares nommées désirs. Elles sont liées au train à grande vitesse (TGV), qui a suscité dans l'hexagone la création de gares bien françaises, comme il ne s'en trouve nulle part chez nos voisins du Nord (l'Espagne, seule, a suivi le modèle français) à savoir des gares hors la ville, gares de plein champ, appelées parfois "gares vertes", peut-être pour céder à la mode du green washing, ou encore "gares bis". À l'intention du voyageur, le sigle TGV est accolé au nom de la ville ou de la région (Avignon TGV, Lorraine TGV) pour bien les distinguer des gares traditionnelles. Le flou de ces appellations révèle donc une forme d'inconsistance. Nous préfèrerons les dénommer "gares exurbanisées" (Troin, 2008).

2 Certes le TGV a également fait émerger des gares urbaines desservant le coeur des villes (Lille-Europe, Lyon Part-Dieu) ou leur périphérie (Massy, Marne-la-Vallée), il a aussi revivifié des gares anciennes (Le Mans, Marseille Saint-Charles), il a enfin créé des garesaéroports (Roissy CDG, Lyon Saint-Exupéry). Nous laisserons de côté ces pôles, relativement intégrés aux territoires urbains (Troin, 1995) pour nous intéresser à cette génération "spontanée" de gares à la campagne.

3 À l'origine de ces implantations, il y a presque toujours les édiles locaux en position d'initiateurs ou d'accompagnants, persuadés que la gare nouvelle leur apportera monts et merveilles. Malgré les déceptions enregistrées, nombre d'élus persistent à vouloir encore aujourd'hui leur gare isolée sur ligne à grande vitesse (LGV). Que signifie cet entêtement? Ces escales de rase campagne, généralement déconnectées du réseau des trains express 
régionaux (TER), flanquées d'immenses parkings ont contribué à désarticuler le maillage des territoires. N'a t-on donc pas retenu la leçon des expériences précédentes? Enfin, n'est-il pas temps d'arrêter cette dilution pour éviter un véritable "désaménagement" de l'espace national (Auphan [1992], avait déjà parlé de "contre-aménagement") et d'envisager la création au niveau central de l'État d'une structure de coordination et de régulation? Nous tenterons de répondre à ces questions.

\section{Les illusions perdues : les gares sur Lignes à Grande Vitesse sont désespérément solitaires}

4 Par édiles locaux, nous entendons les maires, les élus départementaux ou régionaux, les responsables de chambres de commerce et enfin les députés et sénateurs (représentants à Paris de leur circonscription et aptes à faire remonter leurs "désirs localisés" jusqu'au plus haut niveau de l'État grâce au cumul fréquent de leurs mandats). Ils sont ainsi les promoteurs de ces gares nouvelles, reprenant en cela le rôle de leurs aînés qui pesèrent fortement sur les choix d'implantation des gares au cours du XIXe siècle, époque où l'impact des dessertes ferroviaires sur l'urbanisation et le développement économique était cependant tout autre (Merlin, 1991). Certes, ils ne sont pas les seuls acteurs dans ce “jeu” ferroviaire, la SNCF et RFF (Réseau Ferré de France, le gestionnaire des infrastructures) faisant des propositions et arrêtant les décisions après avis de l'État. Mais dans les compromis (par exemple une gare pour deux villes dans le cas de Metz et Nancy), le partage des financements entre pouvoirs locaux et chemins de fer ("nous paierons une partie des coûts de réalisation"), le mixage du technique (une gare de plein champ ne nuit pas à la vitesse élevée souhaitée par l'exploitant pour relier les villes principales entre elles et autorise des dépassements de rames) et du politique ("la gare nouvelle sera le fer de lance de notre développement économique"), les élus locaux ont de grandes responsabilités. La gare exurbanisée peut être acceptée à contrecoeur, faute de pouvoir obtenir un détour des TGV par la gare historique (figure 1), mais elle peut aussi être voulue fortement comme signe de modernité et espoir de renouveau économique. 
Figure 1. Types de situations entre projets RFF/SNCF et souhaits des élus locaux.

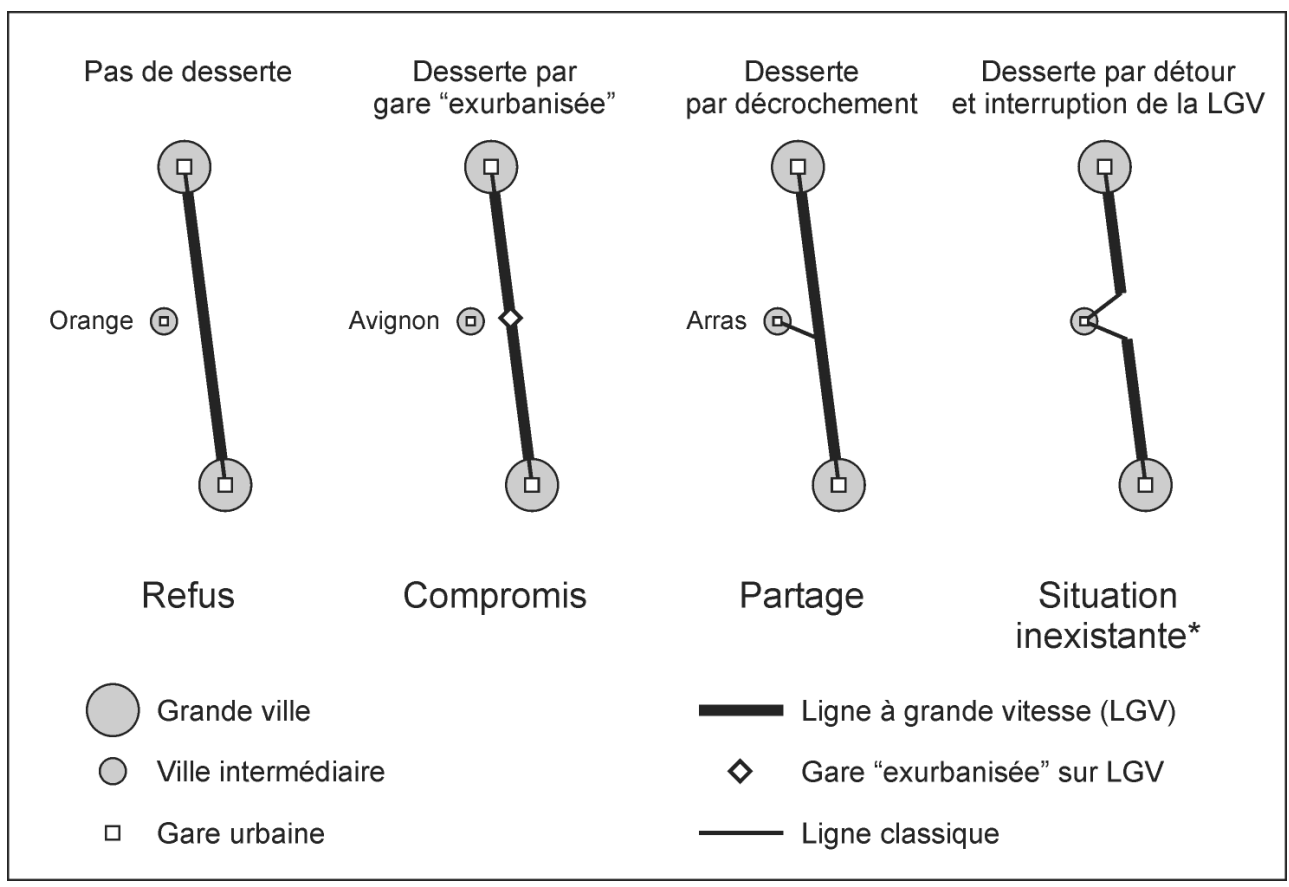

N.B. : la desserte par détour et interruption de la LGV n'existe pas en France à ce jour. Elle est par contre courante dans d'autres pays pour la desserte de grandes villes (Bruxelles, Rotterdam) ou de villes plus petites (Liège).

Le discours des élus évoque souvent, aujourd'hui comme il y a trente ans, la naissance d'activités de production et de services auprès de la gare, le renom et l'image flatteuse dont bénéficiera leur ville, l'extension miraculeuse du bassin de chalandise, voire la génération d'une activité touristique, enfin la facilitation des déplacements vers la capitale. Il faut pourtant reconnaître que la plupart de ces attentes, à l'exception de la dernière, n'ont pas été satisfaites (Ollivro, 1996 ; Plassard, 1992 ; Troin, 2008). Et, isolées, coupées de la ville, même si parfois (Reims, Mâcon) les vagues de la périurbanisation viennent mourir aux portes de la nouvelle gare, nombre de ces stations apparaissent aujourd'hui comme des "sémaphores dans le désert" (Troin, 1998).

En effet, la naissance d'activités nouvelles autour de ces gares hors la ville s'avère être un échec flagrant, et lorsque quelques menues entreprises s'installent à proximité, elles ne doivent rien au TGV, le plus bel exemple étant celui de la gare TGV Haute-Picardie, ironiquement surnommée "la gare des betteraves", où la modeste zone industrielle voisine est née du carrefour routier et autoroutier (noeud constitué par l'A 1, l'A 29 et la N 29) et non pas de l'implantation de la gare TGV (Troin, 2008). Et c'est bien en s'appuyant sur ce carrefour pour automobiles que l'on a justifié la localisation de cette gare TGV sur la commune, jusque là quasiment inconnue, d'Ablaincourt-Pressoir, et non pas quelques kilomètres plus au sud, à Chaulnes, où l'on bénéficiait du croisement avec la voie ferrée Amiens-Saint Quentin. Le drainage routier devait ainsi alimenter la gare en voyageurs et, de fait, il l'alimente (la fréquentation annuelle en 2006 dépassait les 400000 voyageurs), les usagers n'ayant pas d'autre choix que la voiture ou l'autocar pour se rendre à la gare.

De même, dans le cas de Mâcon Loché sur la première LGV française (Paris- Lyon), après une période assez somnolente, les deux zones d'activités situées autour de la gare se sont progressivement remplies, en partie parce que les zones antérieures qui étaient proches 
de l'autoroute $\mathrm{A} 6$ et vouées à la logistique du transport routier étaient saturées. Il y avait là, autour de la gare nouvelle, des réserves foncières encore disponibles et deux zones d'aménagement concerté (ZAC) de 35 hectares abritant 90 entreprises et 800 emplois sont venues donner un semblant d'urbanisation sur les marges de l'agglomération mâconnaise. Urbanisation par juxtaposition et non pas structurée, comme le prouve le fait qu'en 2008, le réseau des lignes régulières de bus urbains n'atteignait toujours pas la gare TGV!

Les promoteurs des gares bis pronostiquaient un meilleur accès au territoire. Encore fautil voir à quelle échelle. Certes, à l'échelle nationale voire internationale, disposer d'une gare TGV “exurbanisée” vaut mieux qu'aucune desserte. Mais à l'échelle régionale, c'est une rupture de charge, via l'automobile, qui est imposée au voyageur. Les élus se flattaient d'une image de modernité : elle peut exister dans le geste architectural de la gare nouvelle ou encore dans la prouesse technologique du TGV, mais cela n'apporte rien à un maillage bien conçu et resserré des territoires et à une bonne synergie des systèmes de transports. La gare TGV "est rarement vue comme point d'entrée dans le réseau régional de transports collectifs" (Zembri, 1993).

Vendôme-Villiers, sur l'axe du TGV Atlantique, en est une parfaite illustration. Voulue avec force par les collectivités locales regroupées dans un Syndicat Intercommunal à Vocation Unique (SIVU) et dans une "Société d'Économie Mixte pour le développement du Vendômois" et arrachée à la SNCF, qui n'y tenait guère, la station a été largement financée par les deniers du Vendômois. Un superbe parc technologique entre gare TGV et ville, sur 40 ha au milieu de boisements de qualité, spécialisé dans les productions liées aux arts graphiques (Vendôme hébergea longtemps l'imprimerie des Presses Universitaires de France et donc la production de la collection Que sais-je ?), était prévu. Un hôtel, un golf, des résidences, devaient s'y ajouter, le tout à 42 minutes de Paris, avec un accès possible par TGV à l'aéroport Roissy Charles De Gaulle. Cet ensemble devait entraîner la création de 1000 emplois dans un bassin de population comptant seulement 65000 habitants. Quinze ans plus tard, il y a bien une gare sur le parc (boisé), mais c'est une gare sans le parc (technologique)! Quelques immeubles de bureaux désespérément vides (à l'exception de rares locaux occupés par des institutions départementales) créent un petit noyau bâti autour de la gare, laquelle offre aujourd'hui six allers-retours quotidiens vers Paris. Mais le flot croissant de voyageurs est avant tout le fait de navetteurs travaillant à Paris, résidant en Vendômois et garnissant pendant la journée de leurs voitures les parkings de la gare qu'il a fallu agrandir. Le pari du développement d'activités et d'emplois sur site était ambitieux et risqué ; il a été perdu et Vendôme est partiellement une ville-dortoir de très grande banlieue, par suite de son accès à la très grande vitesse.

10 Enfin, il faut remarquer, pour relativiser ce soi-disant effet d'entraînement "économique" que les promoteurs de ces gares nouvelles “exurbanisées" n'avaient pas du tout prévu un autre effet, celui-là très négatif : le dépérissement dans la ville proche de la gare centrale et de son quartier commercial. La desserte de ces gares étant diminuée, la gare nouvelle offrant des temps de trajets ferroviaires attractifs, il y a eu déplacement des clientèles et assèchement de trafic et de chalands autour des gares traditionnelles. Valence et Avignon illustrent fort bien cette évolution et chacun peut y constater une certaine décadence du quartier de la gare urbaine avec fermetures de commerces et apparition d'une forme de dépérissement. La gare de périphérie ne deviendra pas pour autant un "centre" 
d'activités et de services, pouvant dédoubler le centre-ville, elle restera marquée par son éloignement urbain et la rupture de charge qu'elle impose (Ollivro, 1996).

11 Ce discours du développement autour de la gare TGV en terre vierge est donc usé, obsolète, dépassé, et même contre-productif. Il nie les acquis de nombreuses études de sciences économiques qui relativisent le lien automatique entre accessibilité et développement économique (Plassard, 1992). Il fait fi des préconisations du récent Grenelle de l'environnement. Il contribue à un éparpillement des pôles de transport. Il ne débouche pas sur une structuration spatiale et territoriale. Et pourtant il perdure !

\section{Partout en France, les projets fleurissent...}

La continuation de ces choix de gares hors la ville est en effet évidente. La figure 2 présente une cartographie combinant les LGV en service, en construction, prévues au plan de relance de 2009 et à horizon plus lointain (2020-2023), d'une part, et les réalisations et projets de gares nouvelles, d'autre part. L'exercice est difficile, car il ne peut atteindre l'exhaustivité, la situation changeant constamment au gré des discussions et compromis dans les collectivités locales, les effets d'annonce venant brouiller les cartes. Nul doute donc que notre document graphique vieillira rapidement.

Figure 2. Les gares nouvelles sur LGV. Situation avril 2010.

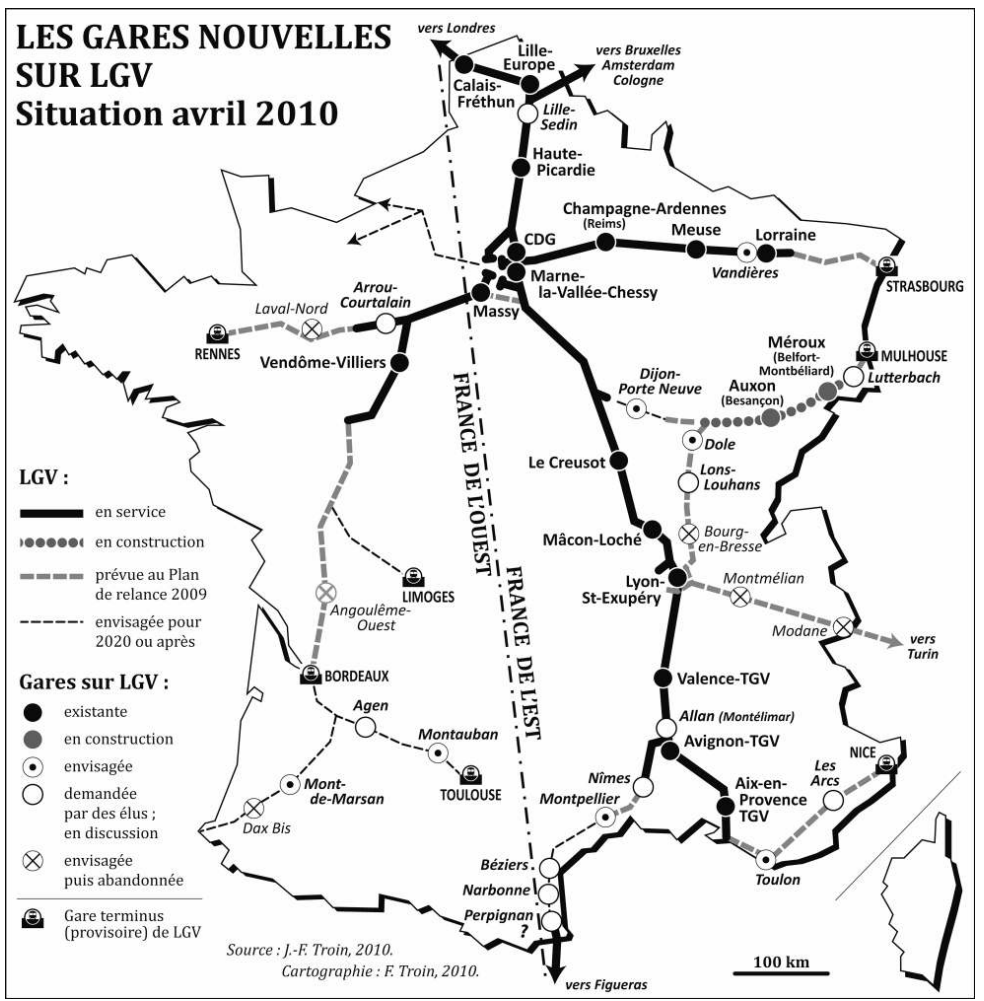

13 L'observation des gares nouvelles sur LGV permet de distinguer :

a. Des gares existantes assez nombreuses sur les LGV Est et Méditerranée

b. Deux gares en construction, à distance de Belfort et Besançon sur la LGV Rhin-Rhône, et devant ouvrir en 2011 en même temps que la première phase de la ligne

c. Des gares envisagées et probables comme Dijon-Porte Neuve, Dole et bien d'autres. À noter le cas spécifique de Vandières, à l'intersection de l'axe ferroviaire de la Moselle sur la LGV Est 
Europe, qui devrait remplacer "Lorraine TGV" construite en rase campagne et aujourd'hui fortement décriée, ce qui laisse une impression de gâchis et provoque des escarmouches politiques à l'approche des élections régionales de 2010, ce qui pose aussi la question de la réutilisation de la gare Lorraine TGV si elle devait être abandonnée : un bel imbroglio!

d. Des gares demandées (désirées) par les élus et parfois aberrantes. rajout, pour combler une lacune vécue comme une offense, et font l'objet de fortes pressions jusqu'aux niveaux élevés de l'État. Ainsi, sur le site d'Arrou - Courtalain sur la branche de LGV Bretagne - Pays de Loire, le député maire de Cloyes (vallée du Loir) caresse le rêve d'une nouvelle gare pouvant desservir Châteaudun (15000 habitants), modeste cité par ailleurs toute proche de la station TGV de Vendôme-Villiers. À quelques kilomètres de Montélimar, un autre élu, à la fois maire, député et ministre ferraille pour une nouvelle implantation à Allan dans une zone de vergers, presque adossée à l'Autoroute du Soleil et qui aurait des effets désastreux sur la gare centrale de Montélimar. Au sud de Lille, un président de conseil régional soutenu par la Chambre de Commerce et d'Industrie, voudrait une troisième gare pour l'agglomération lilloise près de Seclin et de l'aéroport, afin de capter le trafic des Thalys se dirigeant directement vers Bruxelles et qui évitent actuellement les deux gares lilloises, la bifurcation vers la Belgique se situant au sud de Lille. Et bien d'autres exemples pourraient être cités. importe de se positionner dans une compétition interurbaine qui ressemble parfois à une querelle de clochers, mais témoigne cependant bien de l'abandon par l'État des politiques d'"équilibre territorial", exacerbant ainsi la concurrence entre villes ou entre régions et transformant leurs gestionnaires en entrepreneurs publics chargés de capter les investisseurs (voir par exemple Pinson, 1992). Ceci impose de développer des avantages comparatifs parmi lesquels l'accessibilité par TGV ou avion figure souvent en bonne place. Et aussi de partager la poire en deux en choisissant des sites en position intermédiaire entre villes voisines, ce qui reproduit des solutions adoptées au XIX ${ }^{\mathrm{e}}$ siècle sur nombre de voies ferrées rurales. Ainsi, sur la branche Sud du TGV Rhin- Rhône, non encore lancée, est évoquée une gare bressane, entre Lons le Saunier et Louhans, cités qui crieraient évidemment à l'enclavement si une halte salvatrice n'était pas prévue à michemin de leurs territoires respectifs.

Sur le futur axe Nîmes-Perpignan, le dossier du débat public qui vient de s'achever présente, selon les options qui seront adoptées pour les caractéristiques de la future ligne, jusqu'à cinq stations périphériques: Nîmes, Montpellier, Béziers, Narbonne et Perpignan. Certes, à la différence de leurs aînées, certaines seraient en correspondance avec des lignes régionales (TER) et permettraient ainsi une irrigation plus profonde de la région et la possible émergence d'un réseau complémentaire du TGV. Mais combien de dessertes quotidiennes leur seraient affectées? Pour le moment, seule Montpellier TGV semble faire l'unanimité entre Réseau Ferré de France porteur du projet, SNCF et élus. Mais les cités qui font suite sur ce chapelet de villes méditerranéennes (en particulier Nîmes) n'ont pas pour autant baissé les bras et les discussions continuent intensément pour décrocher "la gare périphérique".

17 Sur l'axe Bordeaux-Espagne, dont le calendrier de réalisation est encore bien lointain, la rivalité landaise Dax-Mont-de-Marsan ne semblait pouvoir être apaisée que par la création d'une gare à l'extérieur de chaque ville, d'abord annoncée haut et fort par les élus, lesquels font revivre le temps de Clochemerle à l'heure de la Grande Vitesse. Puis, la 
raison sembla l'emporter : Mont-de-Marsan aurait sa gare périphérique pour des raisons techniques, Dax serait desservie en gare centrale pour des raisons sociales : la première station thermale de France accueille une clientèle âgée et rhumatisante qui utilise beaucoup le train. La gare bis dacquoise refit ensuite un moment surface, comme si on privait la ville de modernité. La gare centrale est pour le moment à nouveau envisagée : est-ce un retour à la raison?

Sur l'axe Bordeaux-Toulouse, au calendrier tout aussi flou, Montauban disposerait d'une gare "exurbanisée". Agen a vu s'affronter les partisans d'une gare centrale dont le réaménagement serait certes coûteux, mais permettrait la réouverture d'une ligne AgenAuch et ceux d'une gare périphérique, venant soutenir un pôle d'activités déjà créé (Agropolis) et bien desservie par l'autoroute. Il semblerait que ces derniers l'aient emporté en utilisant les mêmes arguments qu'en Picardie quinze ans plus tôt !

Les discussions, marchandages et conflits peuvent perdurer et retarder le démarrage des travaux car un levier de chantage existe maintenant: nombre d'élus s'accrochent à leur désir de gare et menacent de ne pas participer au financement de la ligne nouvelle, auquel ils sont aujourd'hui conviés, s'ils n'obtiennent pas la station de leurs rêves. Intérêts locaux et desserte régionale entrent ainsi en confrontation.

Enfin la figure 2 signale des gares un moment envisagées et qui semblent (?) abandonnées aujourd'hui. Bien d'autres phantasmes de gares ont certes existé, mais nous n'avons porté sur la carte que celles qui ont fait l'objet de débats dans les collectivités territoriales ou d'opérations médiatiques importantes.

21 Par opposition, il convient donc de saluer la sagesse des collectivités locales de l'Ouest qui, sur le futur axe Tours-Bordeaux ("Sud Europe Atlantique") tout comme sur la branche Bretagne (Le Mans-Rennes), ont privilégié le passage en gares centrales, ont demandé de multiplier les bretelles de raccordement entre LGV et voies traditionnelles. Le symbole séduisant de la gare flambant neuve en plein champ a été refusé au profit d'une conception plus urbaine du rôle de la gare d'agglomération, dans le cadre de politiques d'urbanisme visant à renforcer les centres-villes et les pôles d'échanges de transports plurimodaux. Cette décision va encore accentuer la forte dissymétrie (indiquée sur la carte) existant entre une France de l'Est, plus densément peuplée et urbanisée où abondent les gares TGV “exurbanisées" et une France de l'Ouest, plus rurale et aux villes espacées où, pour le moment, les gares urbaines sont encore bien desservies par les trains à grande vitesse dans la mesure où ceux-ci circulent le plus souvent sur lignes classiques. Mais la situation pourrait se modifier dans l'avenir (voir Tableau 1). 
Tableau 1. Situation des gares nouvelles sur LGV selon la probabilité de réalisation (en gras, gares localisées dans un tissu urbain péricentral ou en périurbain proche).

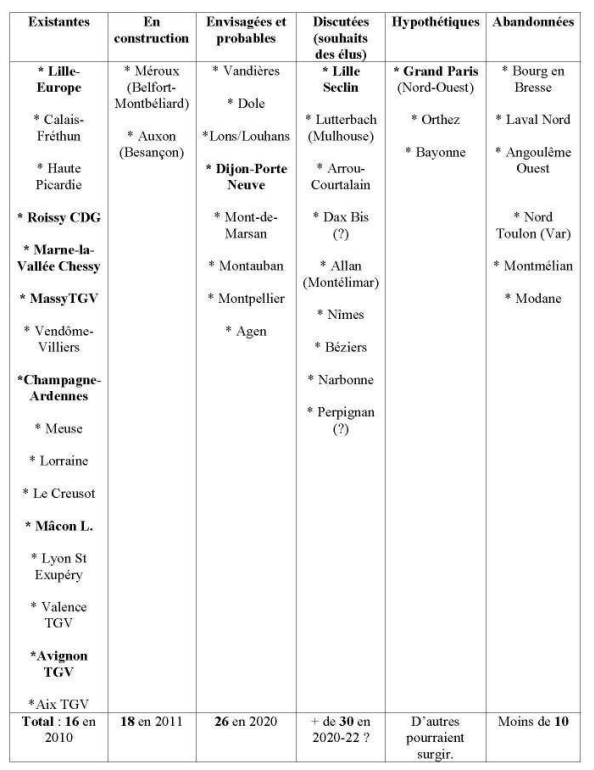

Ce tableau tente d'établir un état des lieux début 2010 et de cerner les probabilités d'existence de gares nouvelles hors la ville dans les années à venir. Ces informations sont fournies sous toutes réserves, nombre de localisations n'étant pas fixées ni figées. Elles démontrent cependant que la tendance à "l'exurbanisation" des gares ne fléchit pas et qu'un revirement en faveur de gares centrales n'est guère perceptible. Si le mouvement continue, c'est une trentaine de gares périphériques qui parsèmeront la France à la fin du premier quart du XXI ${ }^{e}$ siècle.

Raison de plus pour rappeler les combats, pressions, compromis qu'ont connus nos voisins européens pour faire entériner la réalisation de gares TGV dans des stations centrales, alors même que la densité de l'urbanisation et du bâti compliquait sérieusement la réalisation des infrastructures et que des gares de périphérie auraient souvent été moins coûteuses à établir. La centralité a cependant triomphé. Citons ainsi Anvers qui a fini par adopter Anvers-Centraal au lieu d'Anvers-Berchem, au prix d'une lourde transformation de la gare jadis en cul-de-sac et en surface en gare traversante à plusieurs niveaux, plus un tunnel vers le nord de la ville ; Bruxelles-Midi imposée par la Région bruxelloise face à Schaerbeek-Formation un moment envisagée par la SNCB ; Berlin où un nouveau tunnel nord-sud a permis la création d'une gare TGV très centrale, intégrée à la gare Lehrter Stadtbahnhof préexistante, ainsi devenue la principale gare de la ville avec desserte complète par toute la gamme des trains (des trains à grande vitesse aux trains régionaux) et le métro; Liège où les pouvoirs publics ont préféré une reconstruction de la gare existante des Guillemins plutôt qu'une gare nouvelle à Bierset en périphérie; et également Rotterdam ou Barcelone qui ont, elles aussi, lorgné vers des implantations périurbaines et qui ont finalement adopté le passage en coeur de ville au prix de gros travaux. Cette décision se traduit par un allongement du temps de parcours 
ferroviaire, mais elle préserve un accès direct à une multitude de modes de transport de rabattement ou de diffusion au coeur de l'espace urbain et permet ainsi une limitation des circulations et stationnements automobiles pour l'accès à la gare. Il y a là, semble-t-il, des solutions de bon sens que les décideurs français ne semblent pas privilégier. Le cas d'Aix-en-Provence TGV, gare solitaire sur le plateau de l'Arbois est exemplaire: le parking initial de la gare, d'environ 1100 places, a tout simplement été doublé en 2008-2009. C'est la rançon du succès de fréquentation de cette gare nouvelle (2,6 millions de passagers en 2008) ont dit ses promoteurs. Certes, mais nous nous permettrons d'ajouter : c'est aussi une conception assez curieuse du développement durable! Ici aussi, l'implantation de la gare a négligé, voire méprisé une connexion proche avec une voie ferrée transversale (Mannone, 1995) et l'accès à cette "station TGV" ne peut se faire que par le mode routier : le volume des trafics automobiles a donc explosé.

\section{Vers un "désaménagement" de l'espace?} de la ville, tout comme l'ont fait les grandes surfaces commerciales de périphérie, les cinémas multiplex, certaines implantations hôtelières. Le concept de la ville dense qui revient à la mode a été contrecarré par une réalité bien différente, celle de la ville émiettée. Pour entamer un difficile retour à un urbanisme dense, recentré, polyvalent, ne convient-il pas de revenir à des gares de centres-villes, ou éventuellement à des noeuds ferroviaires proches? Le cas de Vandières en Lorraine est typique : il ne s'agit pas d'un site urbain, mais d'un point d'interconnexion donnant accès à un bassin de population d'un million d'habitants, une porte d'entrée à une nébuleuse urbaine, à la différence de Lorraine TGV totalement isolée du territoire (AUT-FNAUT Lorraine, 2008). C'est vers la réalisation de véritables et efficaces interconnexions que les concepteurs devraient s'orienter, en abandonnant ces chimères de gares au milieu de no man's lands, qui veulent singer les aéroports sans en avoir la pertinence. l'accessibilité à la ville, renforcer les pôles d'échanges intra-urbains, choisir le développement centripète plutôt que de renforcer les tendances à l'éclatement ? Finalement, à vouloir toujours gagner de la vitesse, ne risque-t-on pas de perdre du territoire?

Outre qu'on ne peut transformer le TGV en omnibus, desservant un chapelet de gares en positions rurales, ce qui nuit bien évidemment aux destinations lointaines (Givoni, 2006), et que, de toutes façons, ces gares fantasmées ne bénéficieront que de rares arrêts quotidiens, il ne faut pas perdre de vue que c'est en gare centrale que se joue et se pratique l'intermodalité. Réalisée entre train, voitures, tous types de transports urbains collectifs et modes doux, elle est garante d'une pénétration en profondeur de l'espace urbain. Privilégier, sur le mode allemand, les raccordements entre LGV et voies ferrées traditionnelles semble être une solution de raison autorisant une desserte rapprochée des centres-villes et permettant en cas d'incident sur la ligne nouvelle de dévier les convois 
vers les voies traditionnelles. Si, pour cause d'engorgement, la gare historique ne peut plus accepter de trafic supplémentaire, une gare périurbaine reliée par navette ferroviaire ou tramway moderne au centre-ville peut être une solution acceptable. Ce système fonctionne fort bien à Champagne Ardennes TGV (Bezannes) pour atteindre Reims en quelques minutes, en dehors des circulations directes Paris-Reims. La gare de Dijon Porte Neuve, prévue en plein tissu urbain et peu éloignée du coeur de la ville, entre bien dans cette catégorie de stations complémentaires qui sont à la fois un nouvel accès à l'agglomération et un pôle multimodal supplémentaire dans l'espace urbain.

L'intégration forte du réseau TER et des lignes TGV est en effet insuffisamment réalisée aujourd'hui. Elle est, sauf exceptions, ignorée par suite d'un "divorce organisé" entre gares TGV et gares TER (Boudaille, 2002). Elle s'avère pourtant nécessaire, afin que les gares de la grande vitesse ne soient pas uniquement conçues comme des portes d'accès lointaines au territoire urbain, un peu à la manière des aéroports, mais, au contraire via les TER, des instruments efficaces d'une véritable irrigation de tout un arrière-pays. La mise en place plus systématique de TER à grande vitesse (TER - GV) circulant à la fois sur LGV et lignes adjacentes et permettant des temps de trajets interurbains attractifs pourrait être un des éléments de cette intégration. Autour de Barcelone en Espagne, sur la ligne du Kent en Angleterre, ces formes d'hybridation sont admises et plébiscitées par les voyageurs.

Il est assez aberrant que le réseau TGV et le réseau TER soient à ce point déconnectés (sauf rares exceptions), alors que l'une des originalités du TGV est de pouvoir circuler sur tout type de voie électrifiée et que symétriquement des trains régionaux rapides pourraient emprunter partiellement des LGV. Cette continuité de circulation au-delà des LGV sur des parcours terminaux est largement exploitée par la SNCF et donne pleine satisfaction. Mais c'est bien la discontinuité entre les deux réseaux, du fait de la dichotomie gare nouvelle-gare traditionnelle, qui nous prive d'une desserte territoriale plus fine et qui contraint à l'usage massif de la voiture. Et, plus largement, c'est à une "fragmentation de l'espace ferroviaire" que l'on aboutit (Varlet, 1997 et 2008).

La poursuite de ces implantations selon les désirs des élus comporte de réels risques, celui d'une désarticulation dans le réseau ferroviaire, celui d'un démaillage du territoire par un effet de saupoudrage, et finalement celui d'un "désaménagement" de l'espace livré aux caprices de personnalités influentes. Pour éviter ces distorsions, un cadrage national des localisations de gares nouvelles, semblable à ce que réalisait le CIADT (Comité interministériel pour l'aménagement et le développement du territoire) pour les infrastructures linéaires, pourrait être mis en place. Remarquons cependant que le CIADT est devenu CIACT et que les lettres C et T signifient "compétitivité des territoires". Peuton redouter alors que s'exercent concurrences, surenchères et compétitions entre promoteurs de gares créées ex nihilo ? Toujours est-il qu'une coordination/régulation au niveau national s'avère indispensable.

31 En complément et en parallèle, un sérieux recyclage de ces porteurs de projets que sont les élus ou les agents économiques, méconnaissant les bases d'un aménagement raisonné de l'espace et confondant trop souvent dans ces implantations désirées "image" et "fonctionnalité", pourrait être organisé sous forme de sessions de réflexion. En toute modestie, les géographes, entre autres, seraient légitimes pour animer ces sessions et participer à ces exercices pédagogiques. 


\section{Conclusion} par opposition à la plupart des cas étrangers. Une recherche transnationale mêlant taille des villes, organisation des systèmes urbains nationaux (notamment centralisés vs. multipolaires) et modes de gouvernance s'impose probablement, mais dépasse largement le cadre du présent article.

L'auteur remercie vivement Frédéric Dobruszkes pour les compléments au texte qu'il a suggérés et pour la conception de la figure 1.

Le schéma typologique réalisé dans cet article par l'auteur demeure tout à fait valable de nos jours. Le lecteur pourra s'y reporter avec profit. 


\section{BIBLIOGRAPHIE}

AUPHAN E. (1992), “Les gares TGV régionales : un exemple de contre-aménagement du territoire", Hommes et Terres du Nord, 1, Lille, pp. 14-20.

AUT-FNAUT Lorraine (2008), Les raisons de faire Vandières, Dossier par Claude PIERRE DIT BARROIS, aut-fnaut-lorraine.over-blog.com.

BOUDAILLE J. (2002), “Gares TGV : l'usage abusif d'un modèle inadapté”, Le Rail, 91, Paris, pp. 30-33.

GIVONI M. (2006), "Development and Impact of the Modern High-speed Train : A Review", Transport Reviews, 26, 5, September 2006, Routledge, pp. 593-611.

MANNONE V. (1995), L'impact régional du TGV Sud-Est, Thèse de Géographie, Université AixMarseille I, Aix-en-Provence, 567 p.

MERLIN P. (1991), Géographie, économie et planification des transports, Paris, Presses universitaires de France, $472 \mathrm{p}$.

OLLIVRO J. (1996), “L'ambiguïté des gares, clé du développement contemporain”, Les Annales de la Recherche Urbaine, 71, Paris, pp. 35-44.

PINSON D. (1992), Des banlieues et des villes, dérive et eurocompétition, Paris, Les Éditions Ouvrières, $271 \mathrm{p}$.

PLASSARD F. (1992), “L'impact territorial des réseaux à grande vitesse”, in DERYCKE P.H. (sous la dir. de), Espace et dynamiques territoriales, Economica, Paris, pp. 243-262.

TROIN J.F. (1995), Rail et aménagement du territoire. Des héritages aux nouveaux défis, Édisud, Aix-enProvence, $264 \mathrm{p}$.

TROIN J.F. (1998), “Des gares TGV hors la ville : une aberration française”, Urbanisme, 302, Paris, pp. 37-38.

TROIN J.F. (2008), Les gares nouvelles du TGV “exurbanisées”. Fonctionnement et relation au territoire, Rapport de la FNAUT pour la DIACT, Paris, 66 p. Consultable sur Internet : fnaut.asso.fr - Cliquer sur : toute l'actualité, puis dossiers et débats. Ou bien : diact.gouv.fr en précisant gares tgv exurbanisées.

VARLET J. (1997), Dynamiques des réseaux de transport et recompositions territoriales, HDR, Université de Toulouse-Le Mirail, 2 t., 375 et 348 p.

VARLET J. (2008), “Trois révolutions géographiques de la grande vitesse ferroviaire en France", Bulletin de l'Association de Géographes Français, 4, pp. 461-474.

ZEMBRI P. (1993), "TGV et réseau ferré classique : des rendez-vous manqués ?", Annales de Géographie, 571, Armand Colin, Paris, pp. 282-295.

\section{RÉSUMÉS}

Le TGV a suscité en France, à côté de gares urbaines assez bien insérées en ville, des gares “exurbanisées", stations de plein champ, créées de toutes pièces et bien souvent à la demande 
d'élus locaux. Ceux-ci en attendaient de multiples bienfaits dont le développement d'activités auprès de la gare. La plupart de ces attentes n'ont pas été satisfaites. Pourquoi alors existe-t-il toujours un fort "désir de gare" chez des élus locaux, des responsables économiques, voisins d'une ligne à grande vitesse en service ou à construire ? Pourquoi dédaigne-t-on le passage des TGV en gare centrale? Pourquoi ignore-t-on les connexions TGV/trains régionaux pouvant irriguer en profondeur les territoires?

Nous interrogerons l'image perçue du TGV, les compétitions interurbaines, les rationalités économiques, les conceptions territoriales pour rendre compte de ce "désir de gare" et pour conclure à l'impérieuse nécessité d'un cadrage national des rêves d'une grande partie des édiles français, un peu trop prompts à déménager le territoire à leur profit supposé.

The French TGV (High Speed Train), beside urban stations well integrated in the city, created some "out of town" railway stations in the open fields, very often by request of local elected officials. Those officials expected many benefits, particularly the development of activities around the station. Most of these hopes have not been fulfilled. So, why does a strong "desire for a station" still exist among local officials, economy leaders neighbouring a High Speed Line (LGV) in operation or to be constructed? Why is the passing of TGV through central stations so much spurned? Why are connections between regional trains (TER) and high-speed trains (TGV) nearly always ignored?

We shall examine the perceived image of TGV, interurban competitions, economic rationality, territorial concepts in order to account for this "station desire" and to conclude how it is necessary to frame nationally the dreams of a large part of French officials prompt to move the territory for their own supposed advantage.

INDEX

Keywords : high speed lines, new railway stations, accessibility, economic development, networks, local elected officials, town and country planning, France

Mots-clés : lignes à grande vitesse, gares nouvelles, accessibilité, développement économique, réseaux, élus locaux, aménagement du territoire, France

\section{AUTEUR}

\section{JEAN-FRANÇOIS TROIN}

Université de Tours, 29, rue des Trois Tonneaux, F - 37540 St-Cyr-sur-Loire, jf-troin@orange.fr 